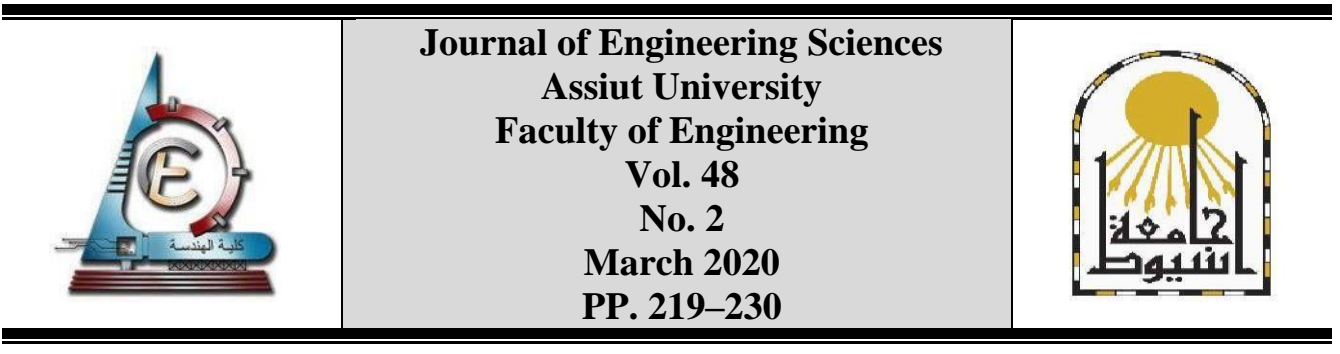

\author{
العلاقة الارتباطية بين معيار الأمن والاحساس بالانتماء:

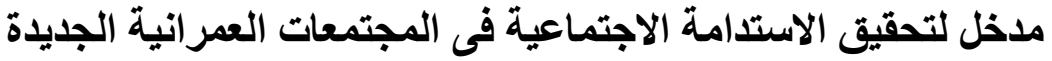 \\ ابتهال جلال الدين محمد ابراهيم \\ قسم الهندسة المعدارية ـ كلية هندسة المطرية - جامعة حلوان
}

Received 2 July 2019; Accepted 27 August 2020

الملخص

تعد الاستدامة الاجتماعية احد ركائز التتمية المستدامة للمجتمعات و التي ادرجت في الدئ الدراسات النظرية و التجريبية

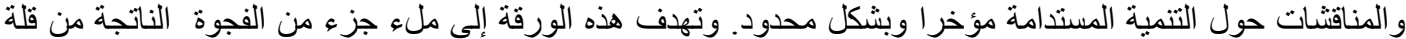

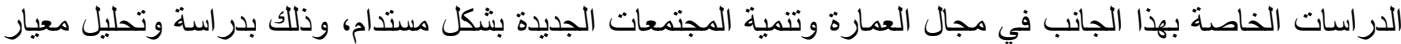

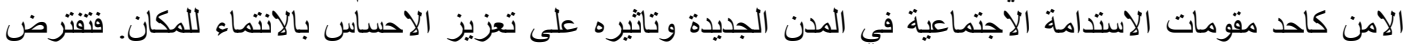

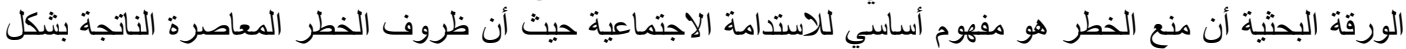

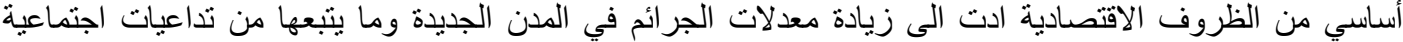

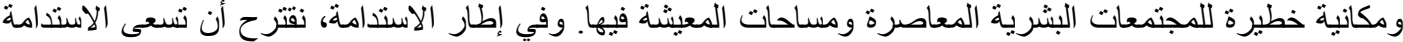

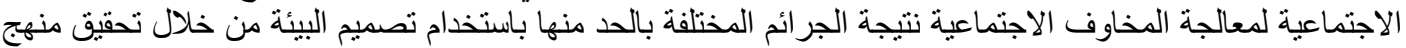

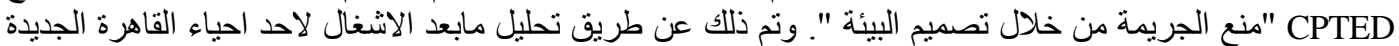

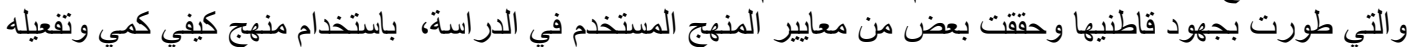

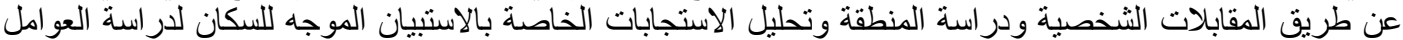

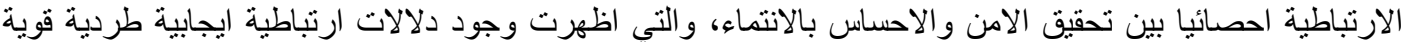

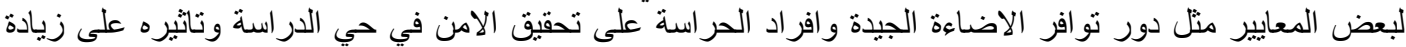

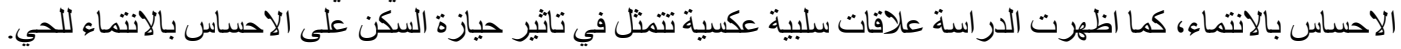

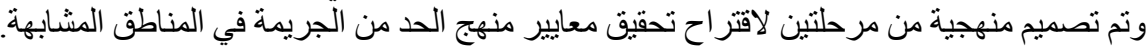

الكلمات الاستدلالية: الاستدامة الاجتماعية، الامن، الانتماء، القاهرة الجديدة،

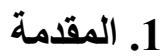

بالرغم من كون الاستدامة الاجتماعية Social Sustainability احد الركائز الثلاثة الرئيسية لتحقيق

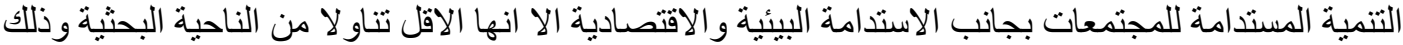

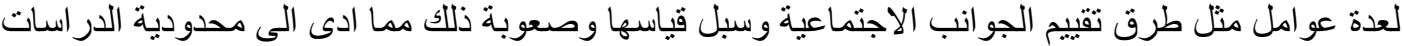

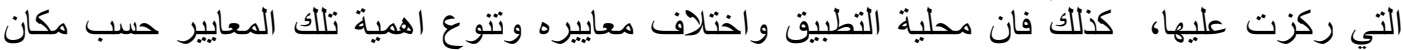

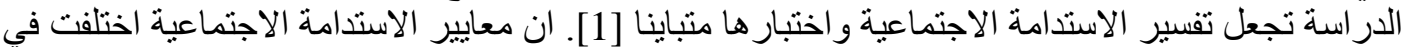

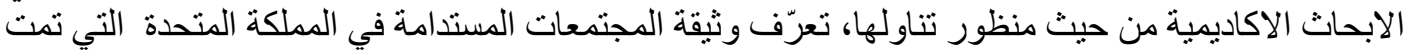

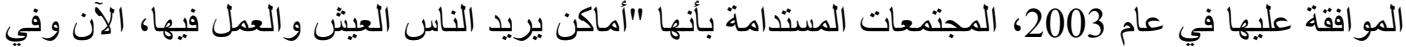
المستقبل. إنها تلبي الاحتياجات المتنو عة للمقيمين الحاليين و المستقبليين، وتكون حساسة لبيئتهم، وتسهر في في

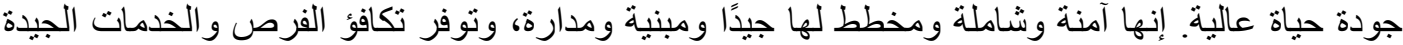
للجميع [2] ". فنشير الدر اسات التي يُنظر فيها إلى مفهوم الاستدامة الاجتماعية من خلال عدسة تصميم 


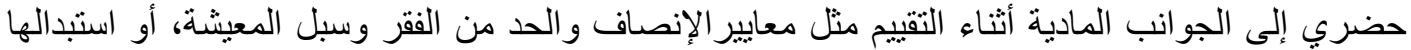

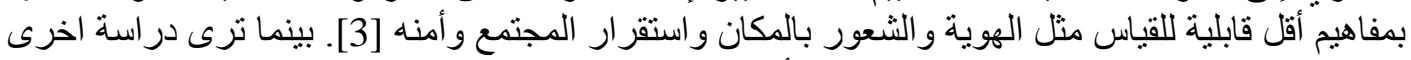

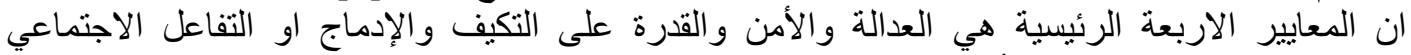

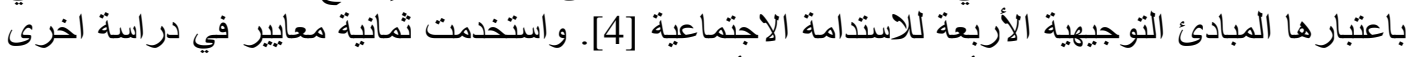

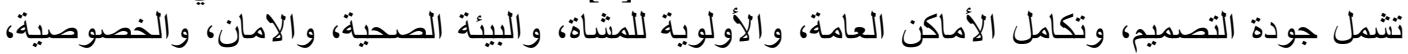

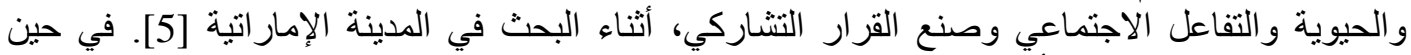

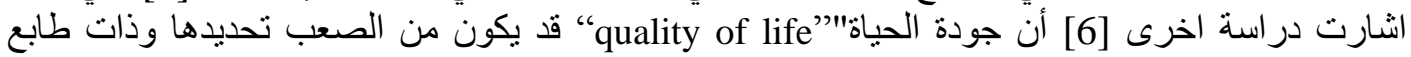
شخصي subjective ولكن هذا الدفهوم يوفر وسيلة للتركيز على البعد "الإنساني" الفعلي للاستدامة التهائ الاجتماعية - حيث يكون الهدف هو تحسين نوعية حياة الناس ومنها تحقيق ما فقد من الامن.

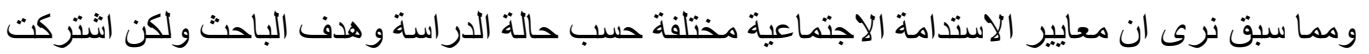

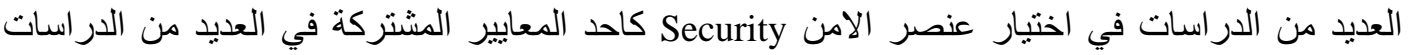

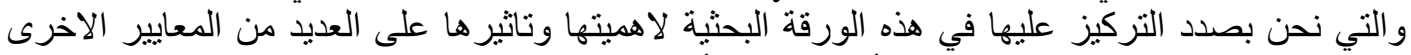

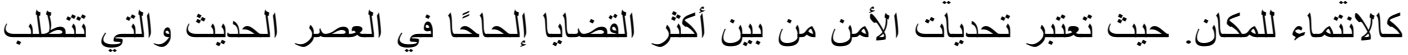

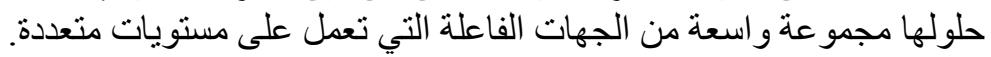

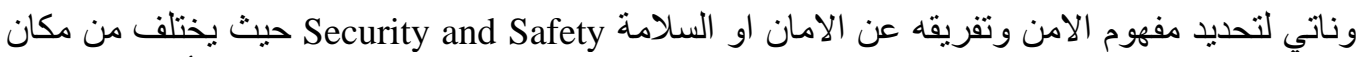

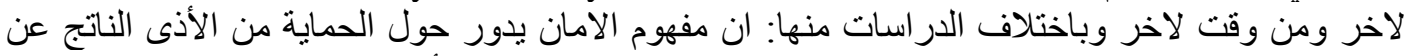

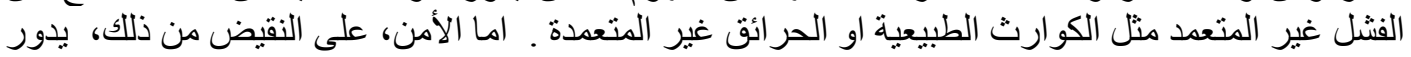

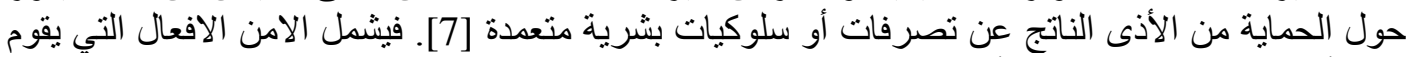

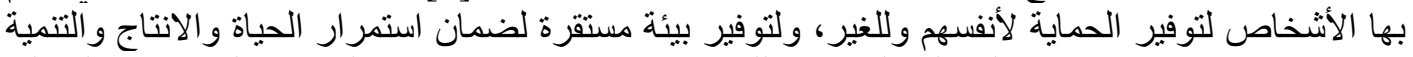

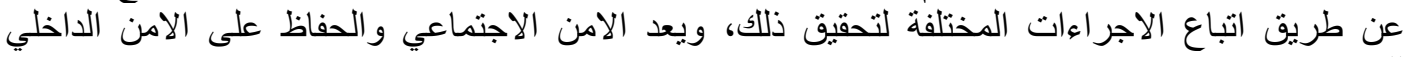

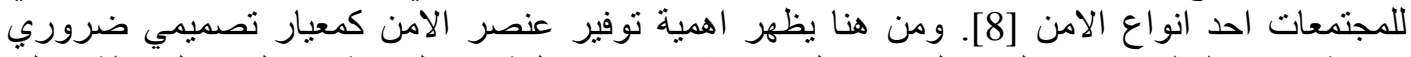

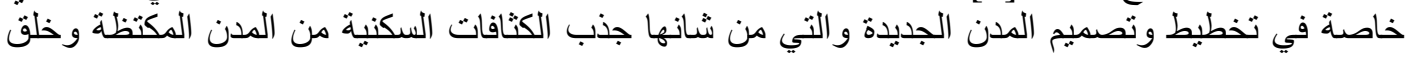

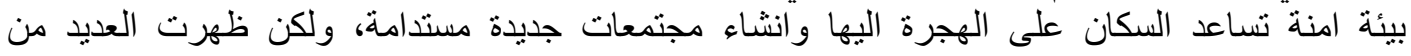

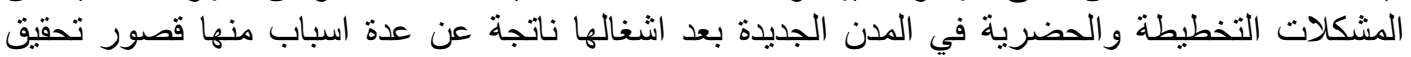

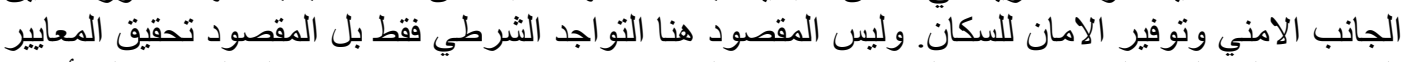

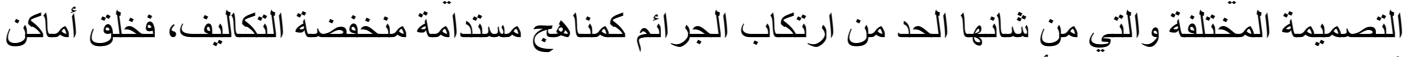

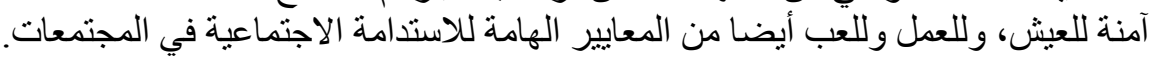

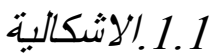

تعتبر المدن الجديدة التى تم تخطيطها لتوفير فرص الإسكان والعمل لسكان المدن المكتظة مر اكز جذب المبان

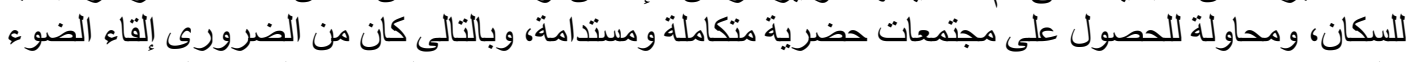

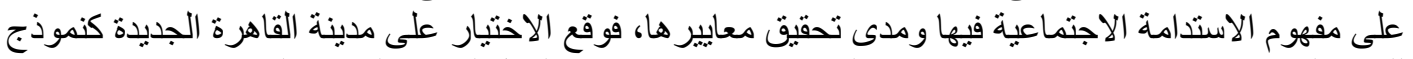

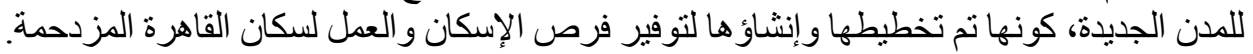
تقدر مساحة مدينة القاهرة الجديدة بنحو 70 ألف فدان، وانشئت بقرار رئاسي رقم (191) لعام 2000

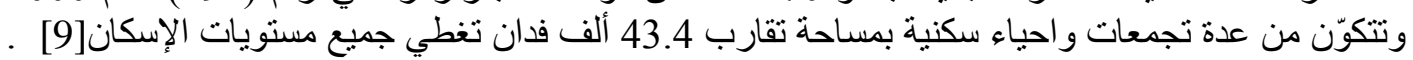

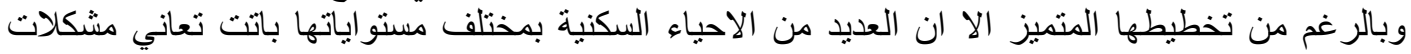

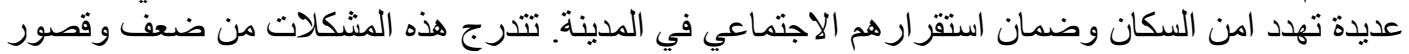

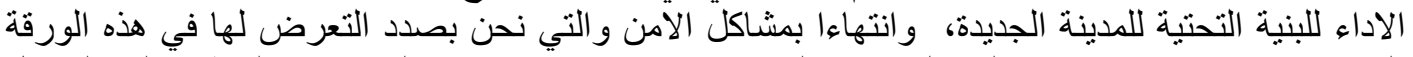

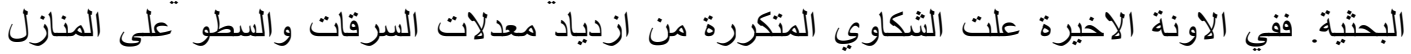

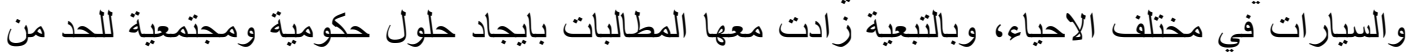

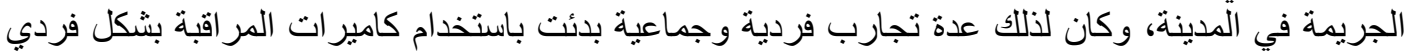
و انتهت بتوظيف شركات امنية خاصة لتامين الاحياء على نفقة السكان. نجحت بعض هذه فئه التجارب بشكل 
جزئي مع استمرار السرقات في المناطق الغير مؤمنة، مما سبب نشر الخوف والفزع بين السكان المقيمين

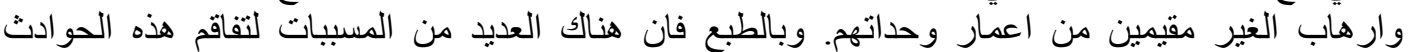

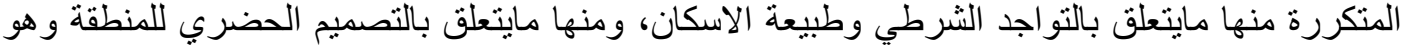

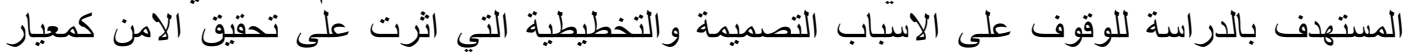

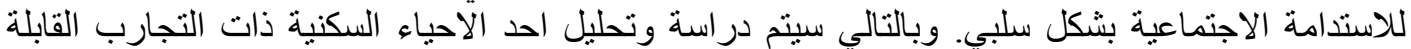

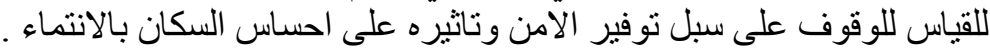

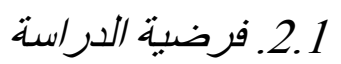

اكدت بعض الدراسات ان الاحساس بالانتماء Sense Of Belonging يعد معيار مهم لتحقيق الاستدامة الاجتماعية، وتفترض الدر اسة وجود دلالات ارتباطية بين معايير تحقيق الامن تصميما من خلال البيئة وبين

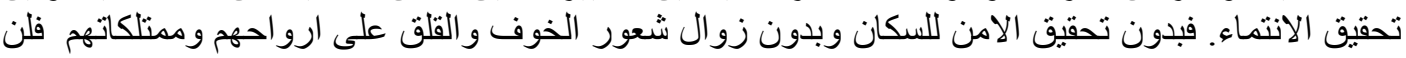

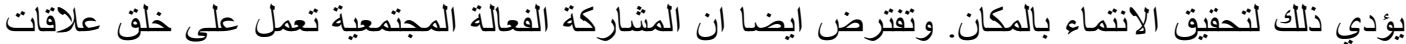

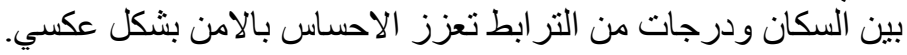

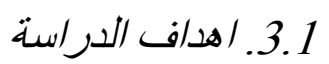

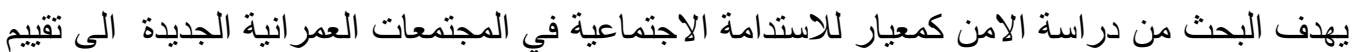

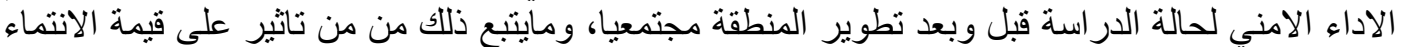

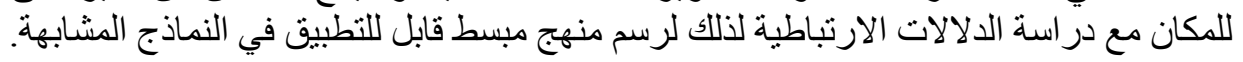

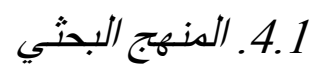

اعتمدت الدر اسة التطبيقية فى منهجيتها على مجموعة من المراحل لكى تحقق الهدف السابق في حالة

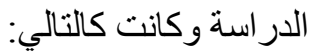

البحث عن نموذج منو افق مع هدف الدر اسة ذو معالم و اضحة قابلة للقياس

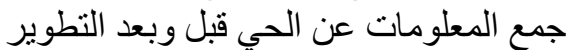

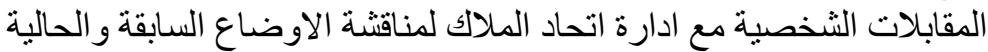

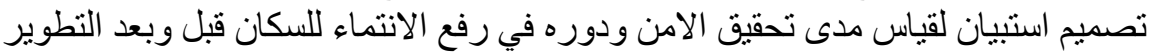

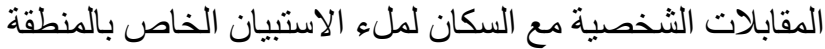

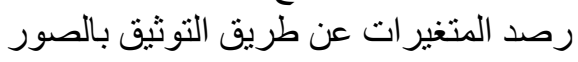
تحليل المقابلات وتفريغ الاستبيان لعمل الدر استة الاحصائية

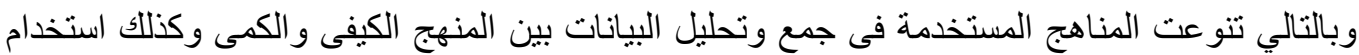

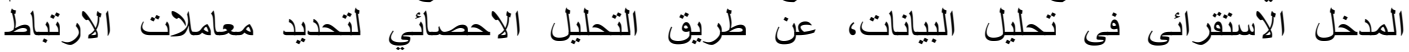
Correlation الجريمة من خلال تصميم البيئة لدر اسة معاييره وتحليله .

\section{2 التعريف بمنطقة الدراسة}

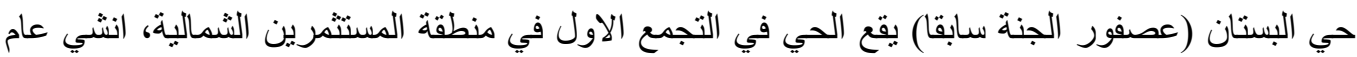

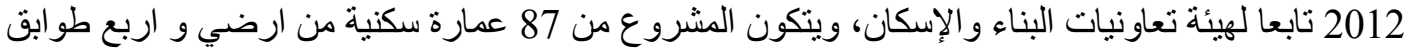

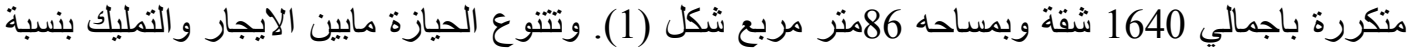

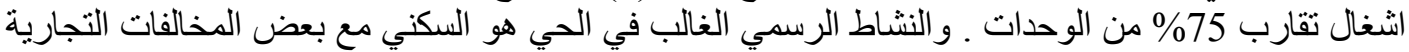

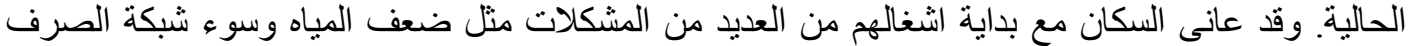
و اتلاف المساحات الخضر اءو والبناء عليها بما يخالف النشاط المحدد للمساحة كمتنفس للسكان، و انتشار القمامة 
اضافة لقطع الانارة الخاصة بالثوارع، مما ادى لظهور المشاكل الامنية كالتحرش والسطو وسرقة السيارات و الوحدات السكنية نتيجة للظلام وتحول المساحات العامة الى مناطق خربة نؤوي الخارجين عن القانون [10].

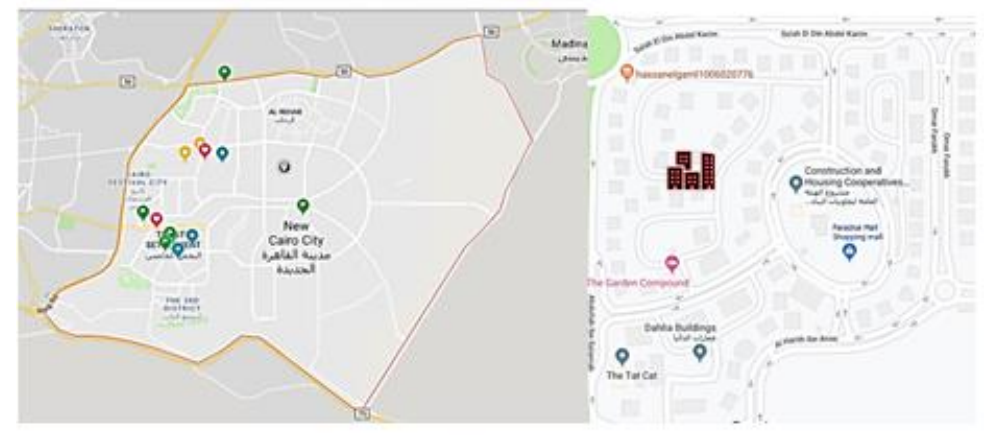

$$
\text { شكل (1): موقع المشروع والموقع العام [11]. }
$$

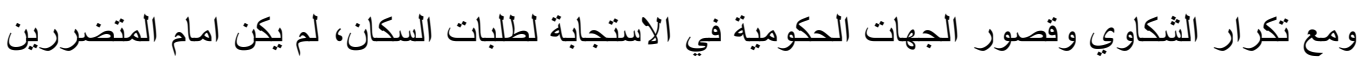

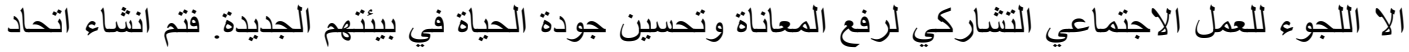

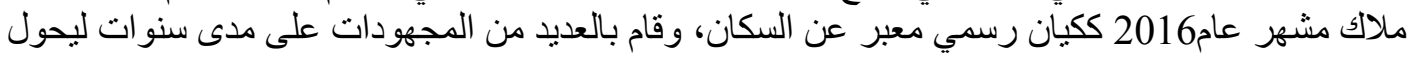
الحي من اسكان تعاوني يتجه للعشو ائية الى تجمي معير عن سكني مغلق.

ولدر اسة الحالة تم عمل لقاءات مع اتحاد الملاك ومع السكان لتسجيل تقييمهم للمنطقة من الناحية الامنية قبل وبعد التطوير وذللك استنادا على بعض فعل معايير منهج (CPTED).

\section{Crime Prevention Through (CPTED) الحد من الجريمة من خلال تصميم البيئة}

Environmental Design

وهو المنهج المنبع في الدر اسة التطبيقية ويعريف منهج منع الجريمة من خلال تصميم البيئة (CPTED)

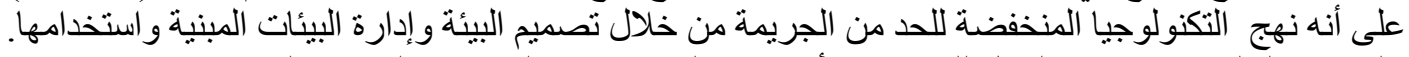

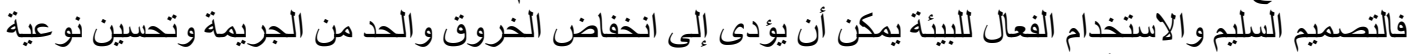

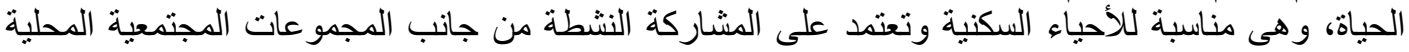

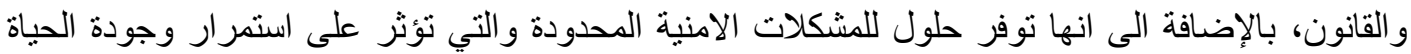

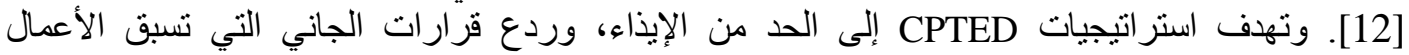

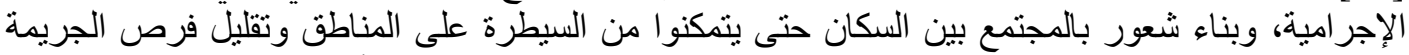

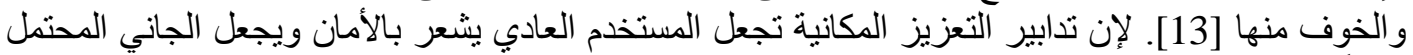

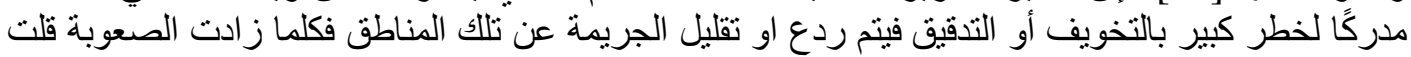

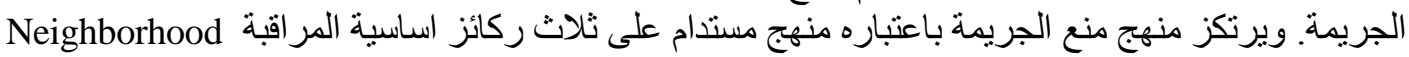
Surveillance ما اعتمدت عليه الدر اسة الاستقصائية والتحليلية وتم تلخيصهم في الجدول التئية التالي:

ويعتبر منع الجريمة من خلال تصميم البيئة (CPTED)أداة مهمة في صندوق الأدوات للمصممين و المطورين لتطوير تجربة المستخدمين و هو نهر نهيم مستدام تجاه التصميم لأنه:

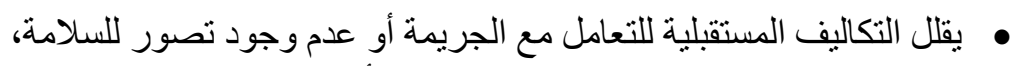

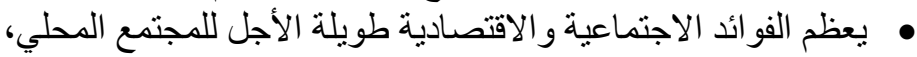

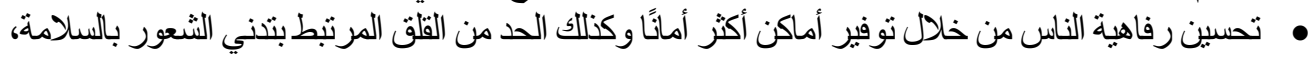
إثر اء الإحساس بالمجتمع عن طريق تحسين التماسك الاجنماعي و الثتعور بالفخر والهوية الثرية المكانية [16]. 
ابتهال جلال الدين محد /بر/هيم ـ العلاقة الارتباطية بين معبار الأمن والاحساس بالانتماء؛ مدخل لتحقيق

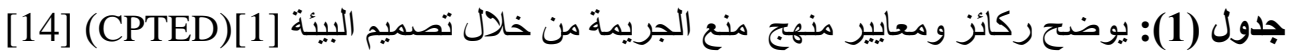

[13] [15]

\begin{tabular}{|c|c|c|c|}
\hline $\begin{array}{l}\text { Reinforcement } \\
\text { Territorial }\end{array}$ & 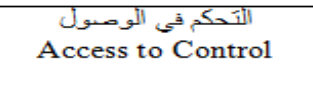 & $\begin{array}{c}\text { المدراقبة } \\
\text { Neighborhood } \\
\text { Surveillance }\end{array}$ & \\
\hline 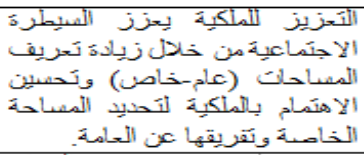 & 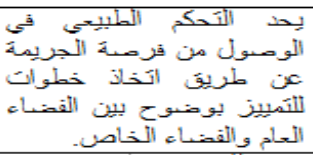 & 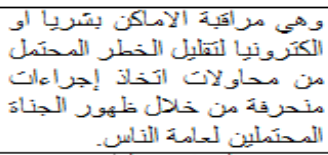 & \\
\hline 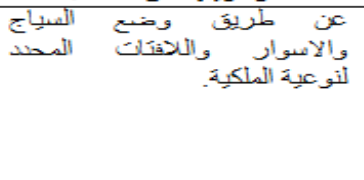 & 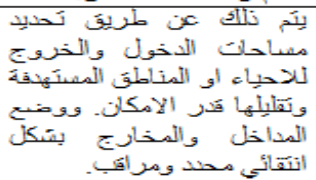 & 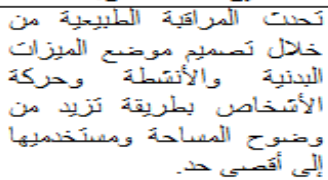 & \\
\hline 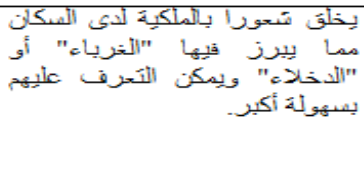 & 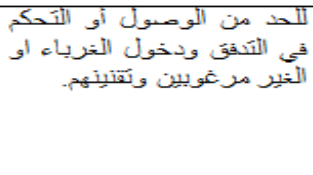 & 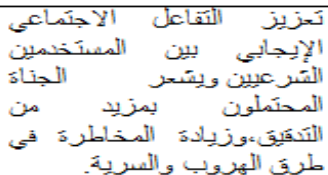 & \\
\hline 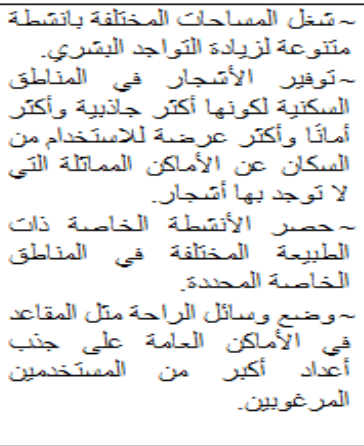 & 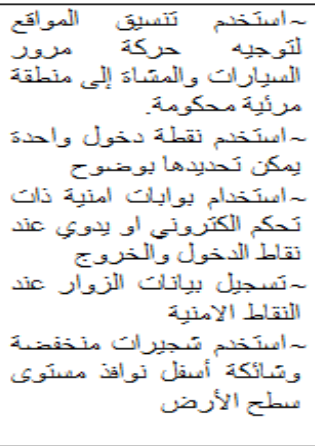 & 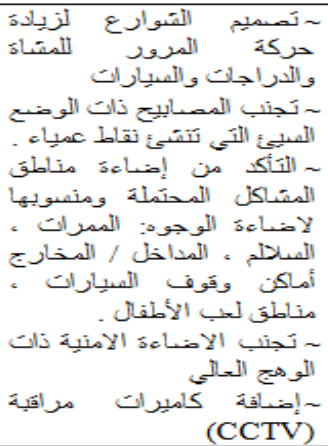 & 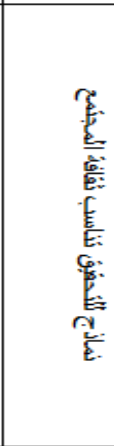 \\
\hline 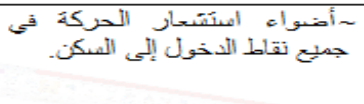 & 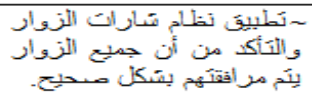 & 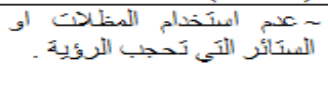 & 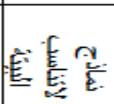 \\
\hline
\end{tabular}

\section{4. الدراسة الميدانية}

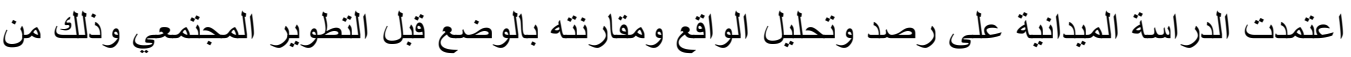

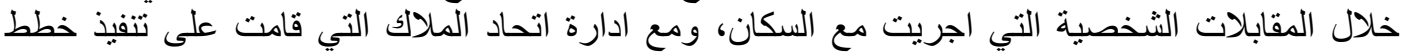

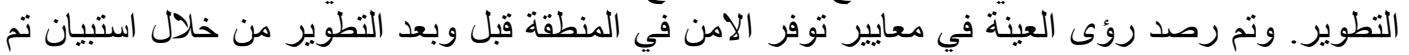

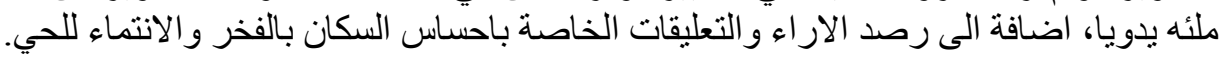
وشملت العينة لقاء 35 فرد من قاطني الحي بنسب قدرت بحوالي: 60\% اناث الى 40\% ذأى ذكور ، تنراوح

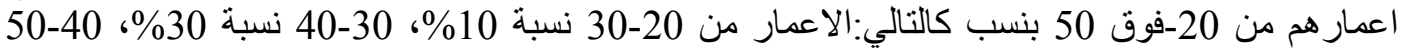

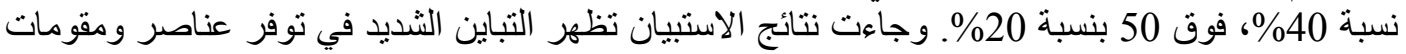

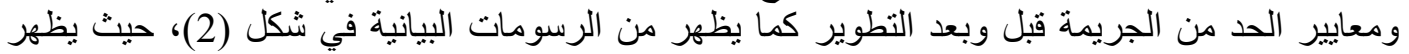

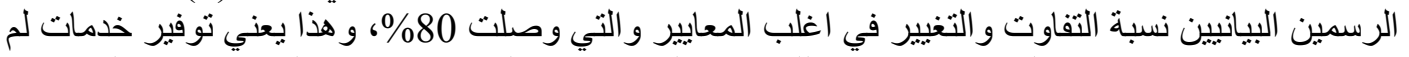

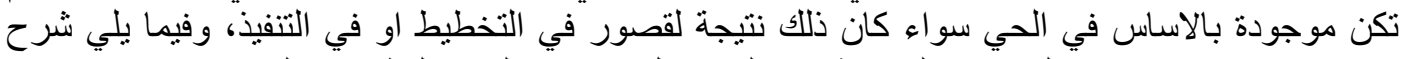

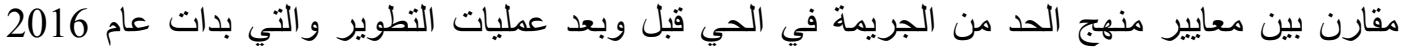

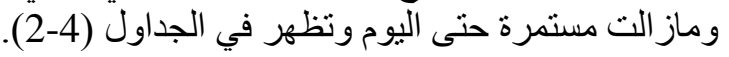



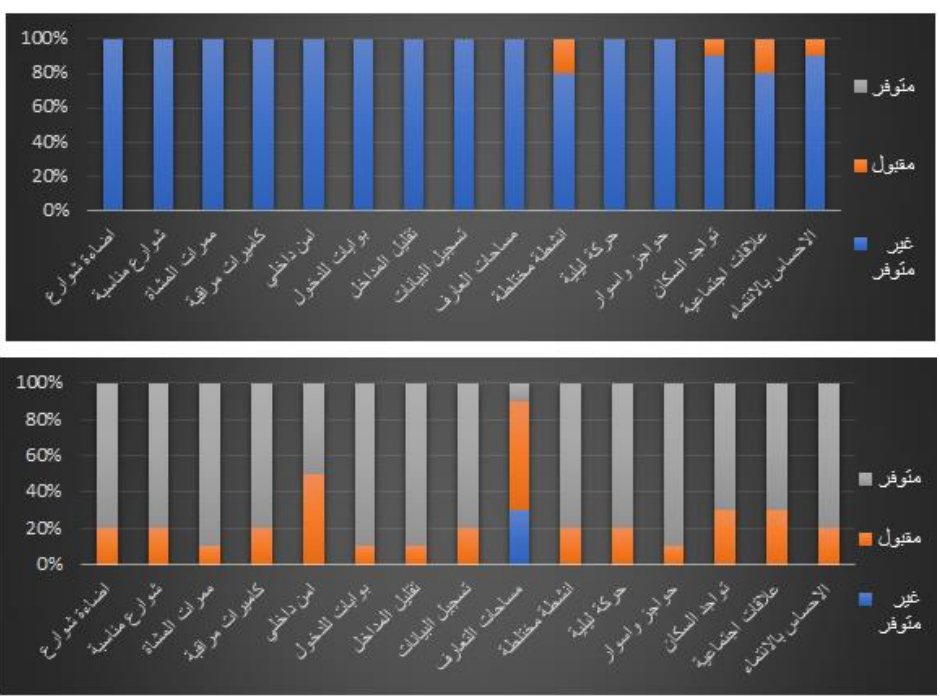

شكل (2): بوضح النسب المؤية لتوفر معايير منهج الحد من الجريمة CPTED قبل(الاعلى) وبعد نطوير الحي (الاسفل) جدول (2): يوضـح الدر اسـة التحليلة للاستبيان الخـاص بمعايير منهج CPTED قبل وبعد تطوير الحي لعناصر المر اقبة يولة مeighborhood Surveillance

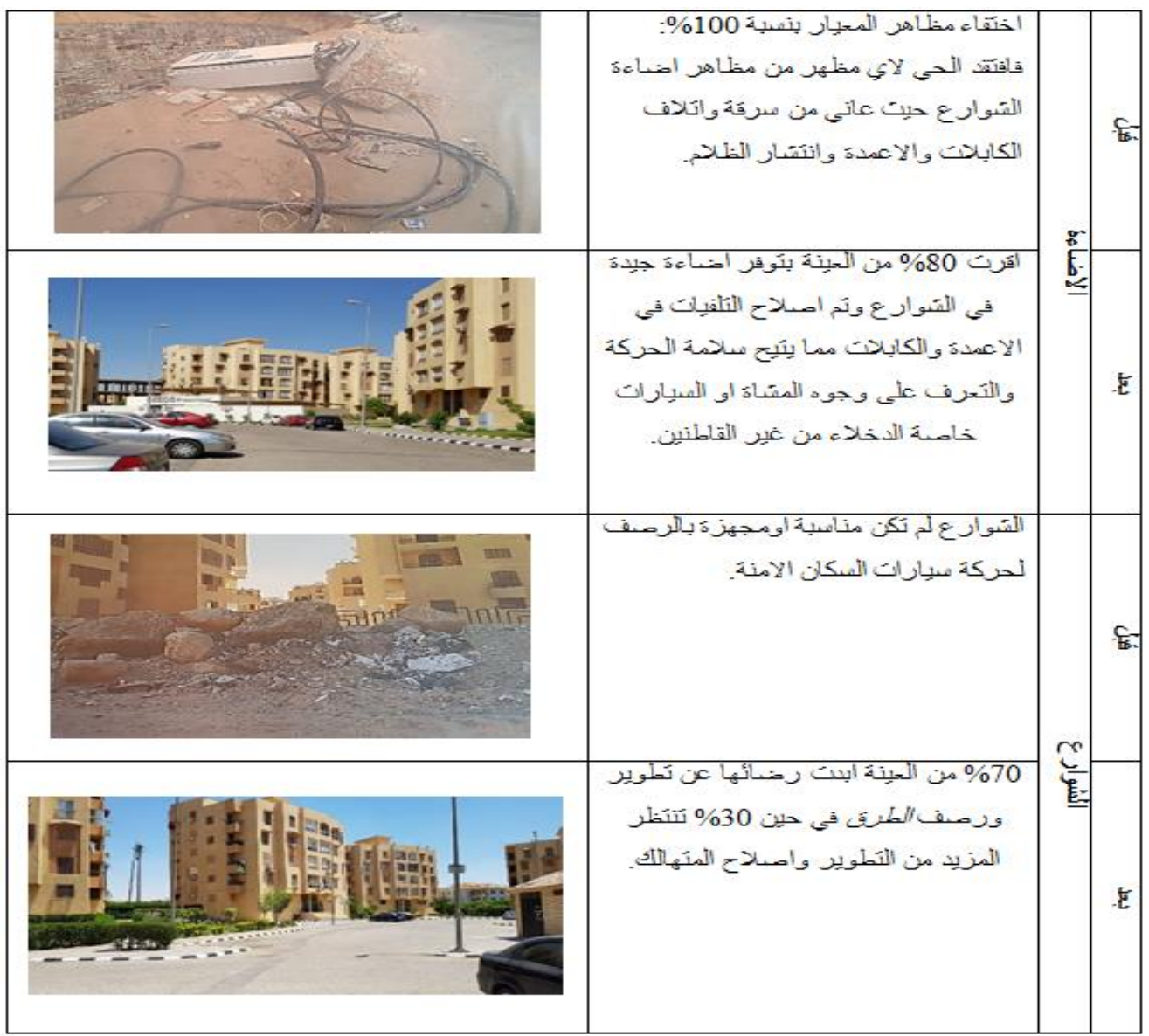


جدول (4): يوضـح الدر اسـة التحليلة للاستبيان الخـاص بمعـايير منهج CPTED قبل وبعد تطوير لحي

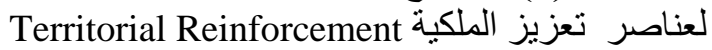

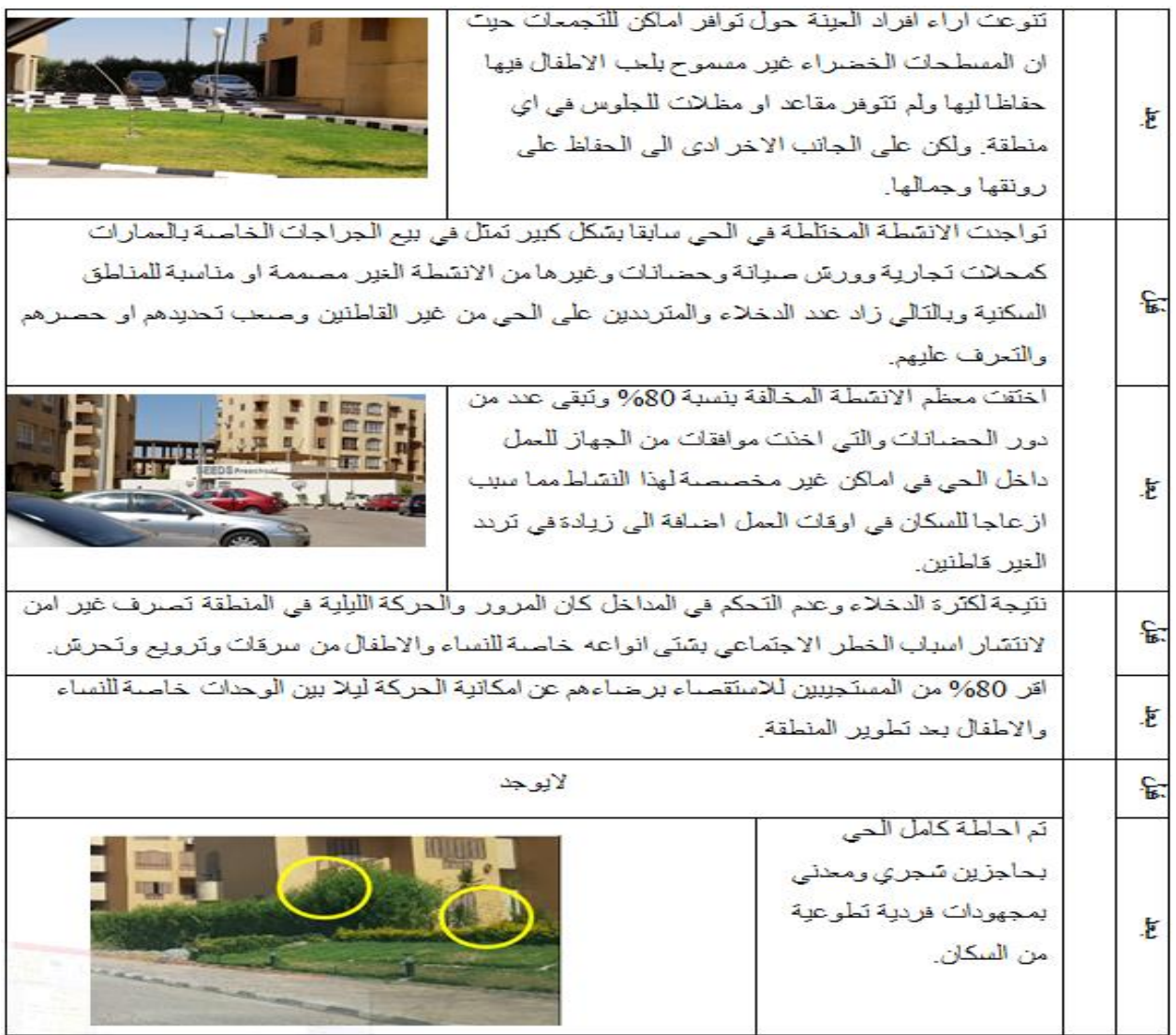

كما عبرت نتايج الاستبيان الى نفور 90\% من العينة من المكان سابقا وعدم وجود اي ارتباط بالحي قبل

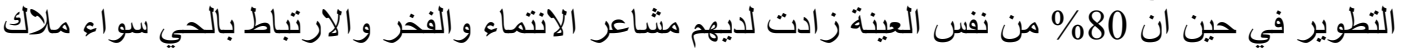

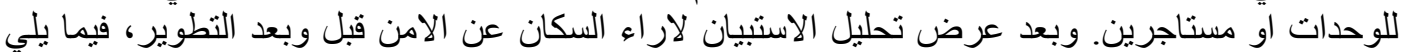
الدر اسة الاحصائية لتحليل تاثير معايير المنهج على الاحساس بالانتماء عن طريق عمل الدلالات الإن الارنباطية

بين المحددات المختلفة جدول (5) .

جدول (5): يوضح الدلالات الارتباطية Correlation بين معايير الامن والاحساس بالانتماء

\begin{tabular}{|c|c|c|c|c|c|c|c|c|c|c|c|c|c|c|c|c|c|}
\hline \multirow[b]{2}{*}{$\begin{array}{c}\overline{7} \\
\frac{7}{7} \\
7 \\
7 \\
7\end{array}$} & \multirow[b]{2}{*}{$\overline{7}$} & \multirow[b]{2}{*}{$\begin{array}{l}\frac{4}{-2} \\
\frac{-3}{3}\end{array}$} & \multicolumn{5}{|c|}{ المر اقكبة } & \multicolumn{3}{|c|}{ اللتحكم في } & \multicolumn{4}{|c|}{ كَزيز الملكية } & \multirow{2}{*}{ 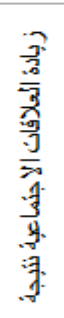 } & \multirow[b]{2}{*}{$\begin{array}{l}\text {-3 } \\
\overline{3} \\
\overline{3}\end{array}$} & \\
\hline & & & $\begin{array}{l}\overline{8} \\
\frac{3}{82}\end{array}$ & 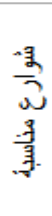 & $\begin{array}{c}\frac{3}{\sqrt[3]{3}} \\
\frac{7}{3} \\
\frac{3}{3}\end{array}$ & $\begin{array}{l}\frac{y}{3} \\
\frac{3}{\sqrt{3}} \\
\frac{3}{9}\end{array}$ & $\frac{\overline{\frac{9}{3}}}{\frac{\sqrt{3}}{3}}$ & $\frac{\overline{3}}{3}$ & $\begin{array}{l}\frac{3}{2} \\
\text {-3 } \\
\text { 浖 }\end{array}$ & $\begin{array}{c}\text { 寻 } \\
-3 \\
3 \\
3\end{array}$ & $\begin{array}{l}\frac{3}{3} \\
\text { - } \\
-3 \\
3 \\
\frac{3}{3}\end{array}$ & 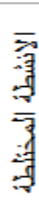 & $\begin{array}{l}\overline{\overline{3}} \\
\overline{5} \\
\overline{\bar{z}} \\
\overline{\bar{z}} \\
\overline{3}\end{array}$ & $\begin{array}{l}\overline{\frac{a}{2}} \\
\frac{2}{2} \\
\frac{2}{2}\end{array}$ & & & \\
\hline ले & $\overrightarrow{0}$ & ᄒᄒ & 당 & $\stackrel{\sim}{\stackrel{c}{i}}$ & $\frac{5}{i}$ & $\stackrel{\overbrace{}}{i}$ & & 잉 & ช้ํㅇ & $\stackrel{\infty}{ٍ ٍ ᄋ}$ & $\dot{i}$ & $\stackrel{\overbrace{}}{8}$ & 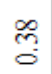 & $\frac{0}{i}$ & : & & \\
\hline
\end{tabular}


ابتهال جلال الدين محد /بر/هيم ـ العلاقة الارتباطية بين معبار الأمن والاحساس بالانتماء؛ مدخل لتحقيق

بعد دراسة وتحليل العلاقات الارتباطية بين معايير منهج الحد من الجريمة CPTED يظهر ان كون كل

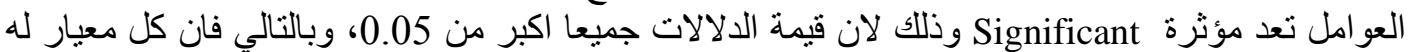

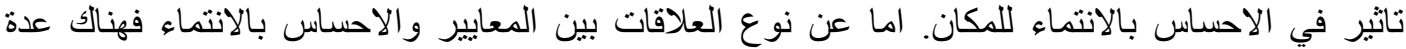

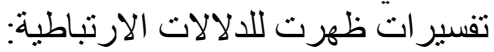

قيم سالبة وتمثل علاقات عكسية و على سبيل المثال لا الحصر :

ـ مثل علاقة توفر شوارع مرصوفة ومناسبة لمرور السيار ات ووجود كامير ات المر اقبة فقد اعطت

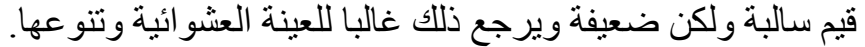

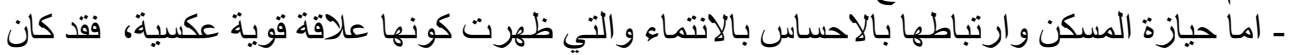

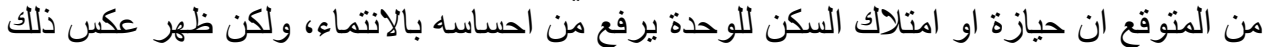

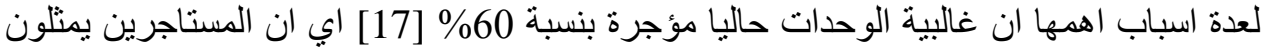

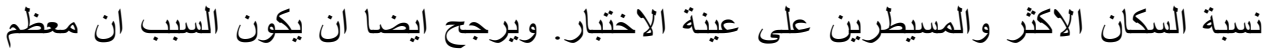

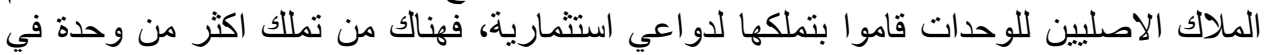

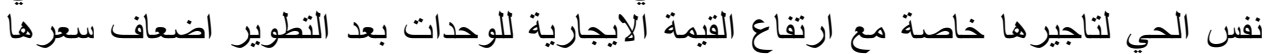

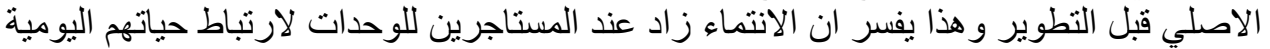
بالمكان مما دعاهم الى حمايته وصيانته و والحفاظ لقئ عليه. قيم موجبة تدل على علاقات طردية ولكن ضعيفة مثال: ـ تسجيل بيانات الزوار على البوابات ويرجع ذلك لعدم تاثر السكان بشكل مباشر بتسجيل بيانات

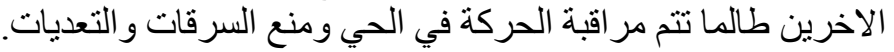

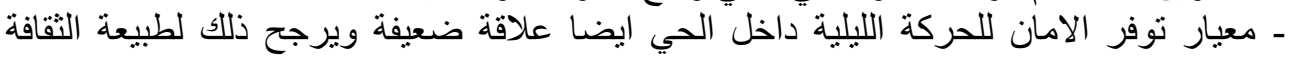

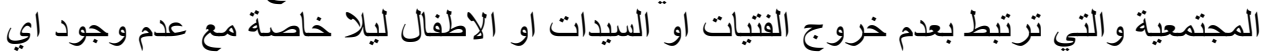

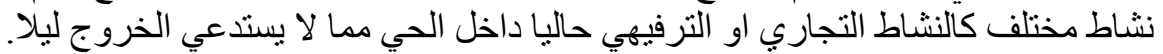

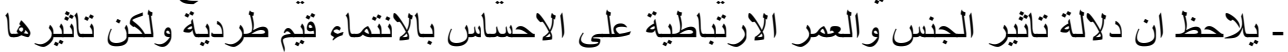
غير قوي في العينة العشو ائية .

اما القيم الموجبة ذات الدلالات الارتباطية القوية الطردية والتي تعلو قيمتها عن 0.5 وتتمثل: ـ في التاثير القوي لوجود افراد امن خاص داخل الحي السكني وليس فقط على البو ابات ما يعطي

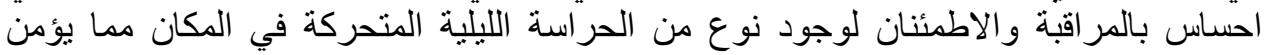

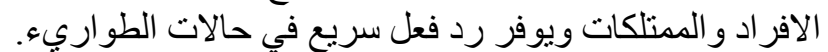

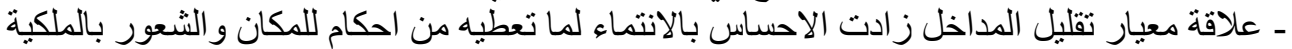

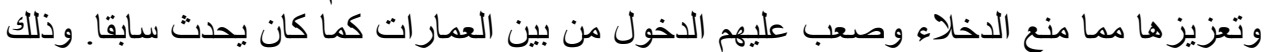

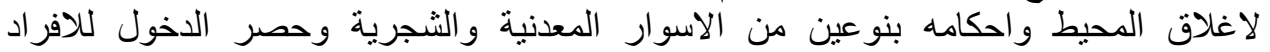
و السيار ات من 3 بو ابات فقط تحت فئ حر استة امنية.

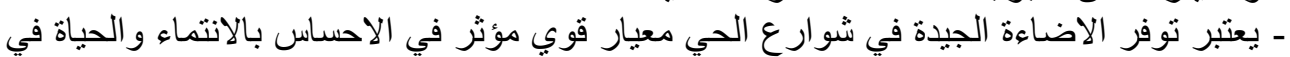

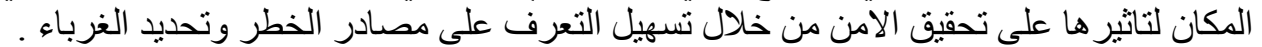

والجدير بالذكر ان عملية التطوير التي حققت بعض معايير الحد من الجريمة قدكانت السبب في وقف التف السرقات بنسبة وصلت الى 100\%، كما اثرت في رفع القيمة الثرائية والايجارية للوحدات السكنية بنسبة

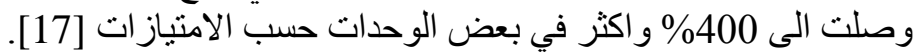

5. 5. 5 التتائج والتوصيات

ان تحقيق الامن في المجتمعات بزيد من الاحساس بالانتماء والذي بدوره يعمل على تقوية العلاقات الاجتماعية لخلق نمط حياة افضل للاجيال الحالية والمستقبلية فيضمن استدامة المكان، وبالتالي هي علاه علاقة 
طردية كلما زادت العلاقات الاجتماعية وقويت زاد الامن والعكس صحيح وينعكس ذللك ايجابا على شعور

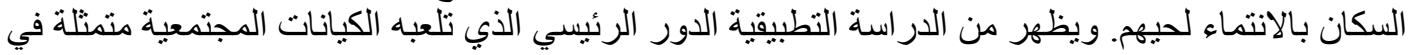
اتحاد السكان/المالك ومدى مساهمته في العملية الانمائية للحي وتحقيق الأمن عن طريق الريقي تطبيق معايير منهج

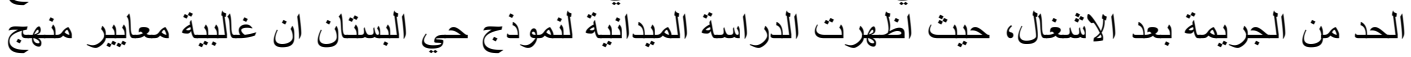

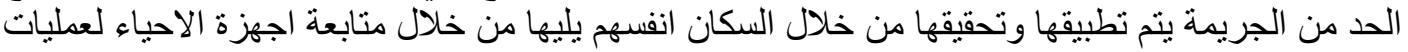

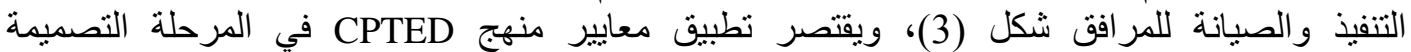

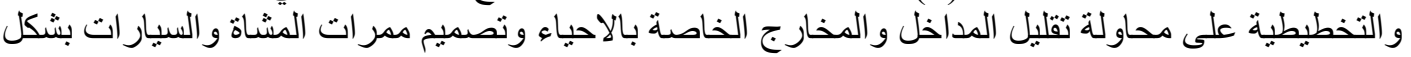
يكثف العبور ويوجهه لمناطق مفتوحة بعناصر تنسيق الموقع (التحكم في الوصول Access to Control وتقليل الانشطة المختلطة (تعزيز الملكية Territorial Reinforcement) -و التي يتم تنفيذها في مرحلة التخطيط ومن خلال اشتر اطات البناء للاحياء- حيث تعد عملية التصميم والتخطيط مرحلة التهلة اولى منتهية. اما

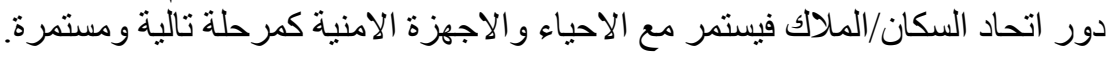

Neighborhood يغطي دور السكان المعايير الثلاثة لمنهج الحد من الجريمة وخاصة (المراقبة (Surveillance الطواريء على مدار ألساعة كمنهجية مبسطة للتفعيل في النماذج المشابهة وفئة

ويعد تحقيق العديد من معايير منهج الحد من الجريمة من خلال تصميم البيئة لنموذج الدراسة بشئ بشكل تلقائي

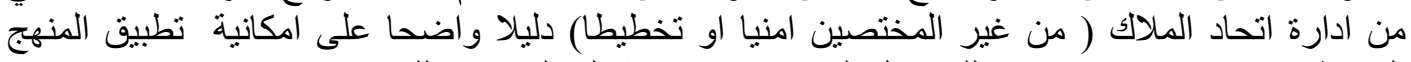
المبسط وتعميمه في باقي الاحياء للوصول التهاد المير توفير الامن بشكل كلي ودائم للسكان.

وفي الختام نؤكد على ان تطوير المجتمعات والمناطق الحضرية خاصة الجديدة منها عملية نشاركية بين

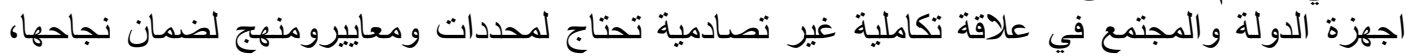

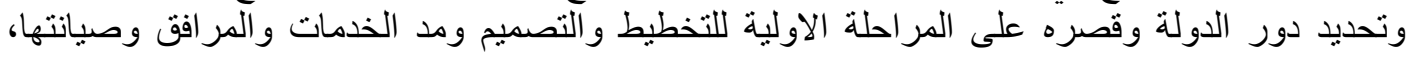

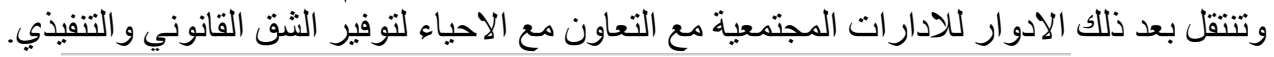

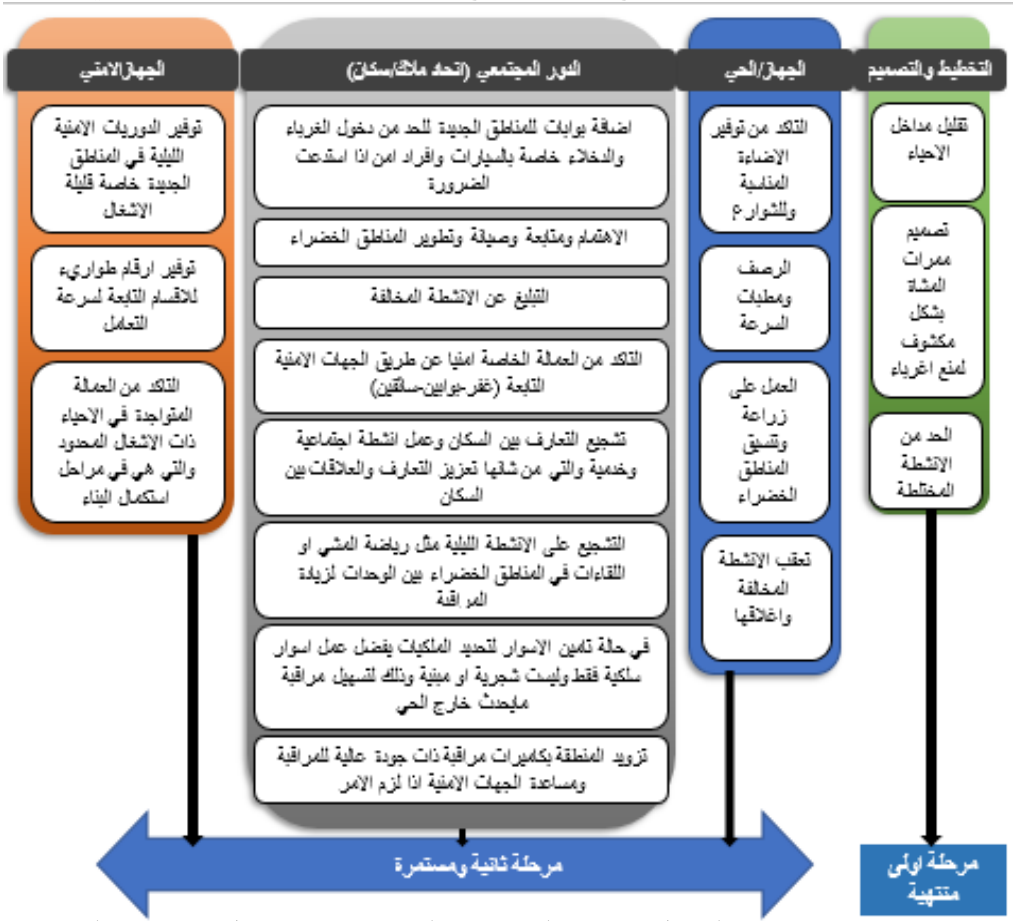

شكل (3): يوضح منهجية مبسطة لتفعيل منهج الحد من الجريمة من خلال تصميم البيئة المختلفة في كل مرحلة بداية من التصميم وحتى مابعد الاشغنال 
ابتهال جلال الدين محدد /بر/هيم ـ العلاقة الارتباطبة بين معبار الأمن والاحساس بالانتماء؛ مدخل لتحقيق ...........

$$
\text { ويخلص البحث للتوصية ب: }
$$

• اعادة النظر في تصميم المناطق الحضرية الجديدة وتفعيل منهج منع الجريمة من خلال تصميم البيئة

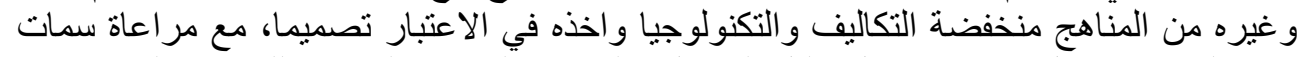

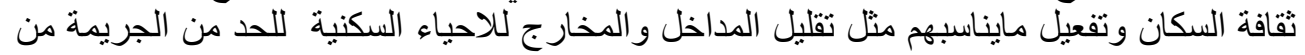

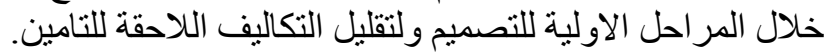

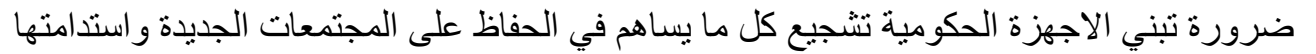

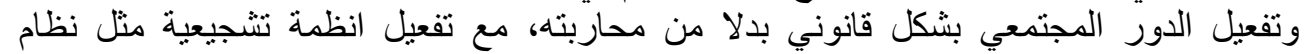

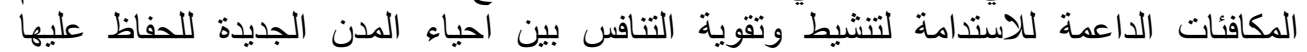

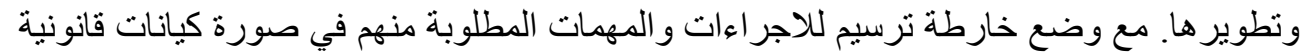

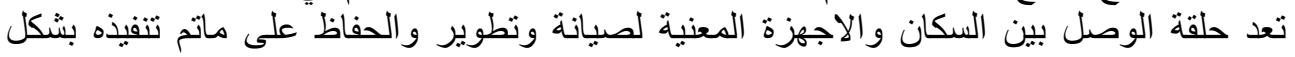
مستدام لتوفير نمط حياة جيد وامن.

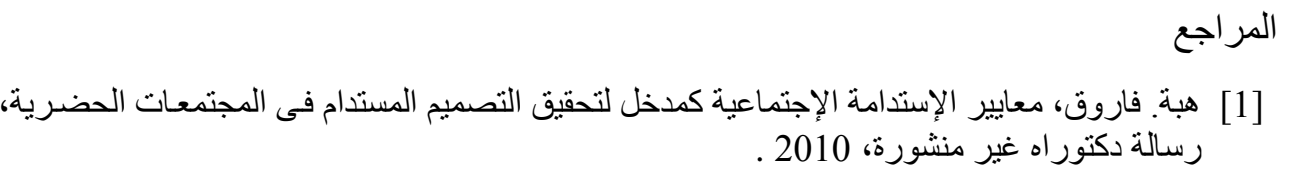

[2] E. Eizenberg and Y. Jabareen, Social Sustainability: A New Conceptual Framework, Haifa: Faculty of Architecture and Town Planning, Technion, Israel Institute of Technology" 2017.

[3] J. \&. W. G. Glasson, "Urban regeneration and impact assessment for social sustainability," Impact Assessment and Project Appraisal, vol. 27, no. 4, pp. 283-290, 2009.

[4] A. S. A. A. S. Ghahramanpouri, "Urban Social Sustainability Contributing Factors inKuala Lampur Streets،" in Asian Conference on Environment-Behaviour Studies, Tehran: 2015.

[5] A. Mehan and F. Soflaei, "Social sustainability in urban context: Concepts، definitions, and principles," in Architectural Research Addressing Societal Challenges, Couceiro da Costa, 2017.

[6] E. Partridge, "Social Sustainability," Sydney, Australia, Institute for Sustainable Futures, University of Technlogy" 2014, pp. 6178-6185.

[7] "Security \& Safety Challenges in a Globalized World," Universiteit Leiden, 2019.

[8] "mawdoo3," [Online]. Available: https://mawdoo3.com. [Accessed 7 2019].

[9] "Maskany," [Online]. Available: http://www.maskany.com/blog/1/post/11. [Accessed 72019 ].

[10] "Almasryalyoum," 2 2016. [Online]. Available:

https://sharek.almasryalyoum.com/cities/cairo/475813/. [Accessed 7 2019].

[11] "Google map," [Online]. [Accessed 7 2019].

[12] R. Wortley and L. Mazerolle, "Environmental Criminology and Crime Analysis," in Crime prevention through environmental design, Willan, Devon (UK), 2008, pp. 153-177.

[13] "cpted," [Online]. Available: .http://www.cpted.net/. [Accessed 7 2019].

[14] "St.Petersburg Police Department," [Online]. Available: https://police.stpete.org/crimeprevention/brochures/cpted-brochure.pdf. [Accessed 7 2019].

[15] "The Balance Small Business," [Online]. Available: https://www.thebalancesmb.com/crimeprevention-through-environmental-design-394571\#territorial-reinforcement. [Accessed 7 2019].

[16] "Auckland design manual," [Online]. Available: http://www.aucklanddesignmanual.co.nz/design-subjects/designsafety/perceptions/guidance/CPTEDintro\#/design-subjects/designsafety/perceptions/guidance/CPTEDintro/whyCPTED. [Accessed 7 2019].

[17 [Interview]. 7 [2019. مقابلة مع ادارة اتحاد ملاك كمبوند البستان/2019] . 


\title{
CORRELATION BETWEEN SECURITY AND SENSE OF BELONGING: AN APPROACH TO SOCIAL SUSTAINABILITY IN NEW URBAN COMMUNITIES
}

\begin{abstract}
Social sustainability is one of the pillars of sustainable development for communities and has been included in recent theoretical and experimental studies and discussions on sustainable development in a limited way. This paper aims to fill part of the gap resulting from the lack of studies on this aspect in the field of architecture and the development of new communities in a sustainable way، by studying and analyzing the standard of security as one of the components of social sustainability in new cities and its impact on enhancing a sense of belonging to the place. The paper assumes that prevention of risk is a fundamental concept of social sustainability، as contemporary risk conditions resulting mainly from economic conditions have led to an increase in crime rates in new cities and the consequent serious social and spatial repercussions of contemporary human societies and areas living in it. As part of sustainability، we suggest that social sustainability seeks to address social concerns as a result of different crimes by using environmental and urban design methodology CPTED "crime prevention through environmental design". This was done by analyzing one of the neighborhoods of New Cairo، which was developed by the efforts of its residents and achieved some of the criteria of CPTED method used in the study، using qualitative، quantitative method، through interviews، study the area and analyze the responses of the questionnaire directed to the population. That done to study coloration that links statistically between the achievement of security and a sense of belonging w which showed the presence of strong positive correlation semantics for some criteria such as the role of street lighting and the guards to achieve security in the case study، and its impact on increasing the sense of belonging c the study also showed negative relationships of housing ownership on the sense of belonging. Finally، the paper suggested simple method to achieve a crime reduction approach in new cities.
\end{abstract}

